\title{
Variability and spectral classification of LMC giants: Results from DENIS and EROS ${ }^{\star, \star \star}$
}

\author{
M.-R. L. Cioni ${ }^{1}$, J.-B. Marquette ${ }^{2}$, C. Loup ${ }^{2}$, M. Azzopardi ${ }^{5}$, H. J. Habing ${ }^{1}$, \\ T. Lasserre ${ }^{4,3}$, and E. Lesquoy ${ }^{4,2}$
}

1 Sterrewacht Leiden, Postbus 9513, 2300 RA Leiden, The Netherlands

2 Institute d'Astrophysique de Paris, CNRS, 98bis Bld. Arago, 75014 Paris, France

3 Max-Planck-Institut für Kernphysik, Postfach 1039 80, 69029 Heidelberg, Germany

4 Departement d'Astrophysique, Physique des Particules, Physique Nucleaire et d'Instrumentation associée (DAPNIA), Service de Physique des Particules (SPP), CEN Saclay, 91191 Gif-sur-Yvette Cedex, France

5 Observatoire de Marseille, LAM, 2 place Le Verrier, 13248 Marseille Cedex 4, France

Received 5 April 2001 / Accepted 6 August 2001

\begin{abstract}
We present the first cross-identifications of sources in the near-infrared DENIS survey and in the microlensing EROS survey in a field of about 0.5 square degrees around the optical center (OC) of the Large Magellanic Cloud. We analyze the photometric history of these stars in the EROS data base and obtain light-curves for about 800 variables. Most of the stars are long period variables (Miras and Semi-Regulars); a few Cepheids are also present. We also present new spectroscopic data on 126 asymptotic giant branch stars in the OC field, 30 previously known and 96 newly discovered by the DENIS survey. The visible spectra are used to assign a carbon- (C-) or oxygen-rich (O-rich) nature to the observed stars on the basis of the presence of molecular bands of $\mathrm{TiO}$, VO, $\mathrm{CN}, \mathrm{C}_{2}$. For the remaining of the stars we used the $\left(J-K_{\mathrm{S}}\right)$ color to determine whether they are O-rich or C-rich. Plotting $\log \left(\right.$ period) versus $K_{\mathrm{S}}$ we find three very distinct period-luminosity relations, mainly populated by Semi-Regular of type a $(S R a), \mathrm{b}(S R b)$ and Mira variables. Carbon-rich stars occupy mostly the upper part of these relations. We find that $65 \%$ of the asymptotic giant branch population are long period variables (LPVs).
\end{abstract}

Key words. stars: evolution - late-type - variables: general - Magellanic Clouds

\section{Introduction}

Stars on the upper asymptotic giant branch (AGB) are often found to be pulsating with large amplitudes and long periods and are classified as: Miras, Semi-Regulars (SRs) and Irregulars. A further characterization in subclasses is problematic. A major discovery by Glass \& Lloyd Evans (1981) is the linear relation between $\log$ (period) $(\log (P))$ and magnitude. Later it was proven that $\mathrm{O}_{-}$ rich and C-rich AGB stars follow the same relation in the $K$ mag (Feast 1989) and in $M_{\text {bol }}$ (Groenewegen \& Whitelock 1996). The $\log (P)$ versus magnitude relation is important because it can be used to measure the distance

Send offprint requests to: M.-R. L. Cioni, e-mail: mrcioni@strw.leidenuniv.nl

* Based on observations collected at the European Southern Observatory.

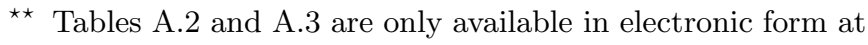
the CDS via anonymous ftp to

cdsarc.u-strasbg.fr (130.79.128.5) or via

http://cdsweb.u-strasbg.fr/cgi-bin/qcat?J/A+A/377/945 of the parent population of these stars independently of other methods. It is also important because stellar pulsation modes are a probe of the internal structure of the stars and allow quantitative estimates of the stellar radius, temperature, density and mass. Two years ago Wood (1999, 2000) revolutionized this picture by showing four relations for LPVs in the same diagram. Wood used data obtained from the MACHO (Alcock et al. 1997) project on the LMC. Very recently the combination of MACHO and ISO data in Baade's window (Alard et al. 2000) in a $\log (P)$-magnitude diagram showed a period-luminosity relation for Miras, but no relation between the period and the magnitude of a large number of SRs. An important aspect of AGB stars concerns their atomic composition. The "third dredge-up", predicted to occur in the thermal-pulsing AGBs (or: TP-AGB phase), changes the surface chemical composition, in particular the carbon surface abundance. The "dredge-up" may turn the star from O-rich into C-rich (Iben \& Renzini 1983). At high luminosities (i.e. $M \approx 5 M_{\odot}$ ) the formation of carbon stars is prevented by the hot bottom burning 
process (Iben \& Renzini 1983). Because the atmospheres of O-rich and C-rich AGB stars are dominated by different molecular bands, the stars with low mass loss rate (such as those discovered by Blanco et al. 1980) occupy statistically two different regions in the color-color diagram $\left(I-J, J-K_{\mathrm{S}}\right)$. O-rich AGB stars have almost all the same $\left(J-K_{\mathrm{S}}\right)$, whereas $(I-J)$ increases with spectral subtype; TiO molecular bands strongly affect the $I$ band Loup et al. (in preparation); C-rich AGB stars have a redder $\left(J-K_{\mathrm{S}}\right)$ due to the effect of the $\mathrm{CN}$ and $\mathrm{C}_{2}$ absorption molecular bands in the $J$ band (Cohen et al. 1981) and the $(I-J)$ ranges from 1 to 2 magnitudes. O-rich stars with high mass-loss rates, $\mathrm{OH} / \mathrm{IR}$ stars will also have large values of $\left(J-K_{\mathrm{S}}\right)$.

In this study we inspected about 800 EROS lightcurves in a region containing the optical center field (OC) in the Bar of the Large Magellanic Cloud (LMC); it is one of the fields searched for AGB stars in the late 1970s by Blanco et al. (1980). Combining these data with the DENIS data we derive three period-luminosity relations. We discuss the properties of the stars in the $\log (P)$-magnitude diagram and in the color-magnitude diagram $\left(J-K_{\mathrm{S}}, K_{\mathrm{S}}\right)$ with the additional information whether the star is C-rich or O-rich. Wood (1999, 2000) found five linear relations, we find only three because of lower sensitivity in our survey data.

Section 2 describes the DENIS data, the EROS data, the cross-identification between these two data sets and the spectroscopic observations. Section 3 describes the classification criteria applied to determine the variability and the spectral type of the sources. Section 4 discusses the period-luminosity relation(s) and the position of different types of variables in the $\left(J-K_{\mathrm{S}}, K_{\mathrm{S}}\right)$ colormagnitude diagram. Finally Sect. 5 summarizes the contents of this study.

\section{Data}

\subsection{DENIS photometry}

From the recently compiled DENIS (Deep Near-Infrared Southern Sky Survey; Epchtein et al. 1999) point source catalogue towards the Magellanic Clouds (DCMC; Cioni et al. 2000a) we extracted all the sources overlapping the selected EROS field (Sect. 2.2). The DENIS observations have been made with the $1 \mathrm{~m}$ ESO telescope at La Silla (Chile) in 1996 simultaneously in the $I(0.8 \mu \mathrm{m})$, $J(1.25 \mu \mathrm{m})$ and $K_{\mathrm{S}}(2.15 \mu \mathrm{m})$ bands; the spatial resolution is $1^{\prime \prime}$ and the limiting magnitudes are 18,16 and 14, respectively. The DCMC contains only sources detected in at least two of three wave bands.

\subsection{EROS photometry}

EROS (Expérience de Recherche d' Objects Sombres) data come from observations carried out at the $1 \mathrm{~m}$ MARLY telescope in La Silla (Chile) between August 1996 and May 1999. A field of $0.7^{\circ}(\alpha) \times 1.4^{\circ}(\delta)$ was simultaneously

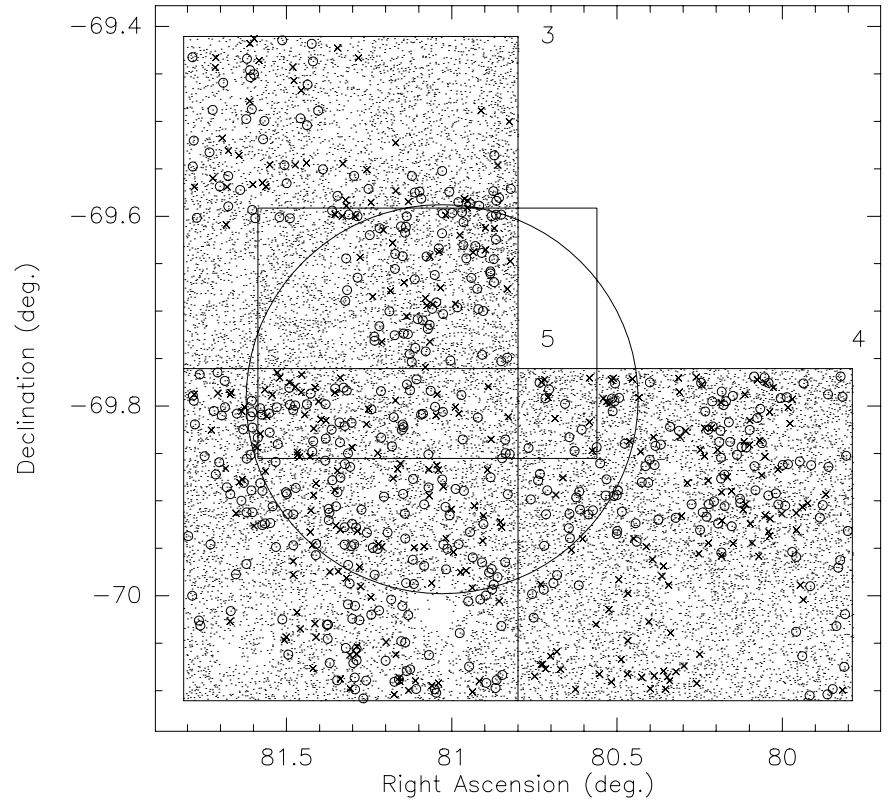

Fig. 1. Map of the analyzed area. The big circle limits the OC field and the rectangle marks the area where multi-object spectroscopy was performed. Small circles identify the classified variables and crosses identify variable stars without assigned classification. Small dots represent all the DCMC sources in the field. The upper right empty area coincides with the position of the out of use CCD. Numbers denote the squared CCDs on the EROS mosaic. The inhomogeneous distribution of the variable stars among the CCD quadrants is due to the incompleteness of the EROS data.

observed in the EROS pass-bands $\left(V_{\mathrm{EROS}}, \lambda=600 \mathrm{~nm}\right.$, $\left.\Delta \lambda=200 \mathrm{~nm}-R_{\text {EROS }}, \lambda=762 \mathrm{~nm}, \Delta \lambda=200 \mathrm{~nm}\right)$. Each EROS field consists of a mosaic of $82 \mathrm{~K} \times 2 \mathrm{~K}$ LORAL CCDs (one is out of use in the red camera) with a pixel size of $0.6^{\prime \prime}$. We used only the central CCDs covering a field of $0.7^{\circ}(\alpha) \times 0.7^{\circ}(\delta)$ square degrees centered on $\alpha=05: 23: 24, \delta=-69: 44: 24$ (J2000). Flat-fielding, bias and dark current subtraction are performed directly at the telescope and the subsequent processing takes place in Lyon (France) at the computing center of the CNRS. For each CCD quadrant a reference image, that slightly overlaps with the images of the adjacent quadrants, is composed. Details about the photometric treatment (PEIDA package) are described by Ansari (1996). The OC field is covered by three CCDs and more specifically by a total of 8 quadrants. Only a small fraction (10\%) of the OC field overlaps with the CCD that is out of use (Fig. 1). Note that due to the poor photometry or to technical problems the detected variables are not homogeneously distributed among the different quadrants.

\subsubsection{Astrometry}

EROS data have not yet been systematically calibrated. We therefore used astrometrically the GAIA/Starlink program and over-plotted the DENIS sources onto the EROS images and applied, in each image, a systematic shift 
until the EROS sources covered a plausible subset of DENIS sources. We tried to use the USNO astrometric catalogue, but in the bar of the LMC the number of available sources is not enough to obtain a convergent solution. Therefore we used all DENIS sources detected in three bands and obtained quickly the shift that provided a very good coincidence between the two catalogues. We found only a few double cross-identifications (two EROS candidates for the same DENIS source); in these cases we inspected the corresponding images and selected the closer candidate.

\subsubsection{Light-curves}

For each cross-identified source we extracted a lightcurve. Next we searched each of these light-curve for periods between 1.5 and 1000 days. The combined extraction and search program is based on a method developed by Schwarzenberg-Czerny (1999) and calculates two magnitudes $\left(R_{\text {EROS }}\right.$ and $\left.V_{\text {EROS }}\right)$ and few other parameters. One parameter, " $q$ " denotes the quality of the variability; only sources with $q>25$ are considered to be variable. Sources with $q>50$ have generally a "good-quality" light-curve and sources with $25<q<50$ are clearly variable but their light curve is rarely good enough to determine any periodicity; we call these light curves of "acceptable-quality". In our sample 743 sources are variable of which 444 have good-quality and 299 acceptable quality. Aliasing does not affect the extracted light-curves. About 60 sources of the good-quality show clearly two simultaneous periods. A separate detailed analysis, on all variable sources, where first the dominant period is derived and then the residuals are used to search for other periods is now being performed (Verschuren et al. in preparation).

\subsection{Optical spectroscopy}

We used the ESO $3.5 \mathrm{~m}$ New Technology Telescope (NTT) at La Silla (Chile) on the nights of November 17 to 19, 1998 to obtain low-resolution $(R=270)$ spectra between $\lambda 3850-10000 \AA$. A sample of 143 AGB stars in the OC field in the Bar of the LMC was selected from the DENIS color-magnitude diagram. For comparison we added 31 objects of known spectral type (Blanco et al. 1980).

We used the Multi-Object-Spectroscopy mode of the EMMI instrument. We observed six overlapping fields of $5^{\prime} \times 8^{\prime}$ with on average 14 slits punched per mask. The CCD frames were corrected for the bias level introduced by detector electronics (a set of 9 frames per night were observed and then combined to calculate the statistical median bias value for the whole period), for the dark current (no dark frames were observed during the same period so we used a value of $0.03 \mathrm{e}^{-} / \mathrm{pix} / \mathrm{min}$ determined during another observing campaign; the stability and the almost negligible value of the dark signal is confirmed by the NTT - team) and for the relative pixel response (flat-field - three frames were observed and mediated per mask). The sky-subtracted spectra were then wavelength calibrated and corrected for the atmospheric extinction. The data reduction process was done using the MIDAS package.

Five of the observed spectra are of poor quality, but good enough to determine the $\mathrm{C}$-rich or $\mathrm{O}$-rich nature of the corresponding source. 14 of the observed spectra are useless because the star was too faint or because of electronic interferences. In one case the wrong star was observed and in two cases the slit was in between two blended sources. In the end we confirmed the spectral type of 30 objects and assigned the spectral type to 96 objects of the original sample (Sect. 4). Note that at the position of BMB-O 46 (Blanco et al. 1980) we detected a star of $\mathrm{C}$ type while Blanco et al. assign it a late $\mathrm{M}$ type.

\section{Classification of the light-curves}

With the data provided by EROS we derive high-quality light-curves for sources covering almost the entire Cloud regions and with a sampling in time that was previously only possible for very short period variables. The sensitivity of the EROS instrument allows amplitudes as small as $\Delta I=0.1 \mathrm{mag}$ to be measured, a factor 3 better than in previous monitoring campaigns by i.e. Hughes (1989). The increased accuracy of the light-curves also improves the classification of the curve. In the GCVS Kholopov (1985) gives the following criteria: - Miras have well pronounced periodicity in the range $80-1000$ and a large amplitude $(\Delta V>2.5)$; - Semi-Regulars of type a (SRa) have persistent periodicity showing an amplitude smaller than Miras; (amplitude and light-curve shape usually vary and the period is in the range $35-1200$ days), - Semi-Regulars of type b $(S R b)$ have poorly expressed periodicity (mean cycles in the range 20-2300 days) or with alternating intervals of periodic and slow irregular changes, and intervals of constant magnitude; two or more periods may be simultaneously present.

Frequent sampling is essential to discover irregularities or to confirm regularities in the shape of the light curve. For example the distinction between $S R a$ and $S R b$ focuses on the regularity of the periodicity and on the presence of more than one period, which can only be disentangled when the sampling of the light-curve is frequent enough. These better sampled light curves allow a much better application of the classification criteria introduced in the GCVS to a much larger sample of stars.

So far the detection of small-amplitude variables in the Magellanic Clouds has been limited. The large survey by Hughes (1989) detected only variables with $\Delta I>0.5$ mag. Detailed light curves from observations of variables in our Galaxy have been preferentially determined for large amplitude variable stars or stars with infrared excess.

There are many more variable stars observed by EROS that can be classified with refined criteria and considerably better statistical completeness. Such studies are outside of the scope of this study. 
(a)
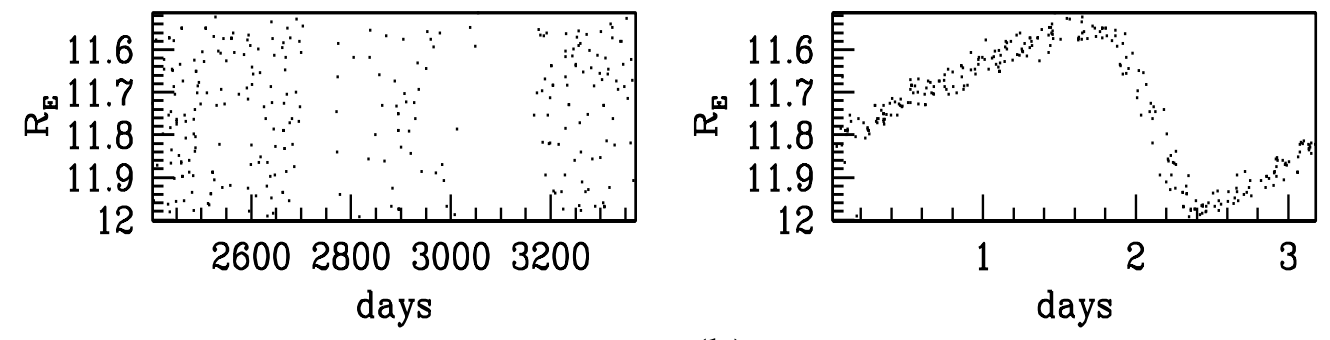

(b)
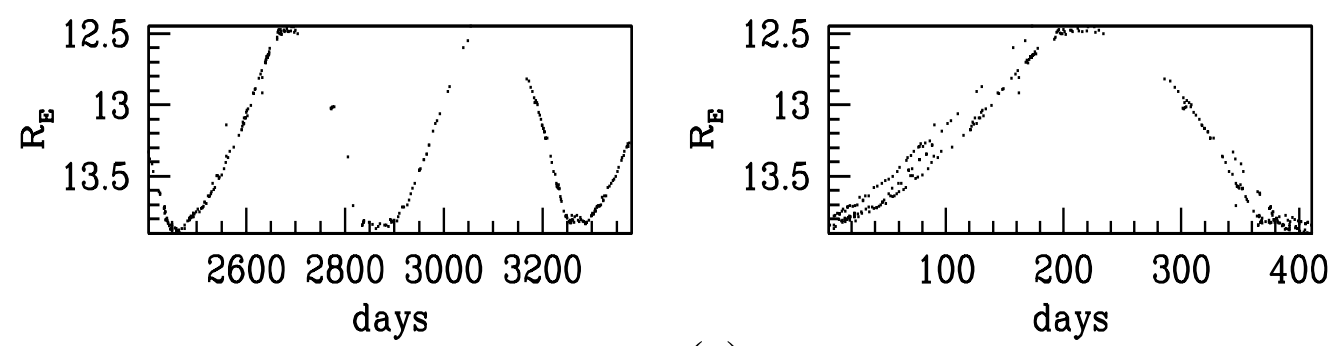

(c)
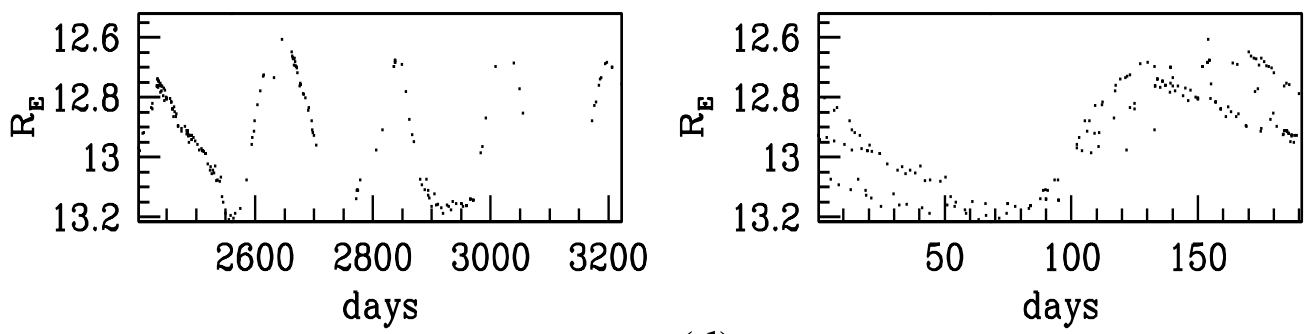

(d)
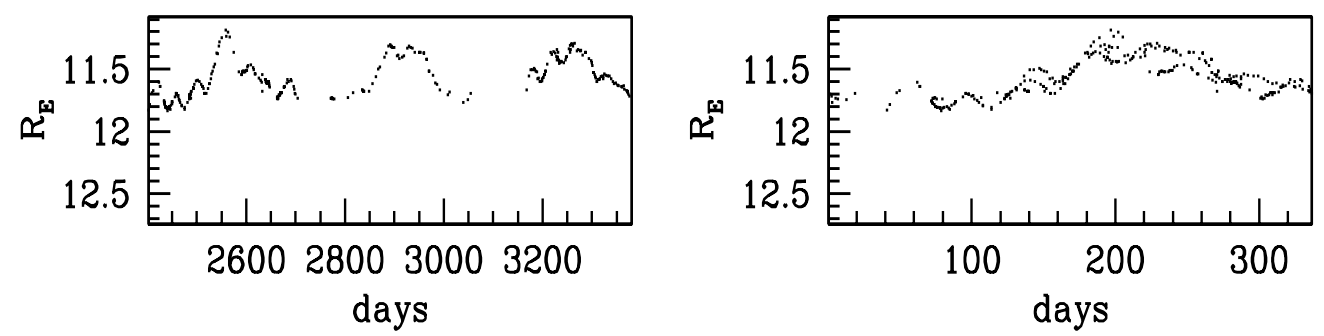

Fig. 2. Light-curves in the red EROS filter over the measured time range (left) and folded on the major period (right) for: a) cepheids, b) Miras, c) $S R a$ s and d) $S R b$ s. Origin of days for left panels is January 1st, 1990 (JD 2447892.5 ).

\subsection{Cepheid variables}

Cepheids (Fig. 2a) have periods from 1 to 50 days but some larger periods have also been found. We found 80 Cepheids $(7.5 \%$ of our total number of variable DENIS stars). Most are detected by DENIS only in the $I$ and $J$ bands; their color is blue reflecting the warm temperature of the photosphere $(I-J \approx 0.7)$. In this paper we concentrate on LPVs and so we do not further investigate Cepheids.

\subsection{Long period variables}

Mira variables (Fig. 2b) are AGB stars of spectral type M, $\mathrm{C}$ or S. They have a stable period that falls in the range from 80 to 1000 days; their amplitude exceeds 2.5 mag in the $V$ band, $0.8 \mathrm{mag}$ in the $I$ band (Eggen 1975) and about $0.5 \mathrm{mag}$ in the near-infrared. The pulsation is poorly explained: the pulsation mode (fundamental, first or higher overtone) is not yet established definitively, nor is the connection between mass-loss and other physical parameters.

We classify 43 sources as Mira variables adopting the criterion that $\Delta R_{\mathrm{EROS}}>0.9$, these sources amounting to $8 \%$ of the AGB stars (Fig. 2b). Their light curve may show the following additional characteristics: - maxima variable by up to $1 \mathrm{mag}$ - small variation of the period from cycle to cycle - presence of a bump in the ascending or descending branch - variation of the phase of minimum. Variables with smaller amplitude of variation have been classified as $S R$. We identify $25 \%$ and $6 \%$ of AGB stars as 
Table 1. Percentages of AGB stars as discussed in the text.

\begin{tabular}{lr}
\hline Type & $\%$ \\
\hline Mira & 8 \\
$S R a$ & 25 \\
$S R b$ & 6 \\
$S R$ & 6 \\
acc. quality & 20 \\
non-variable & 35 \\
\hline
\end{tabular}

$S R a$ (Fig. 2c) and $S R b$ (Fig. 2d), respectively. $S R b$ s have more than one pulsation period. Six $\%$ of the AGB stars have a light-curve with too much structure to identify them with either of the two sub-classes of $S R \mathrm{~s}$. Among the acceptable-quality light curves there are sources which can be classified as "irregular variables": clearly variable but irregular in amplitude, shape and size of the individual peaks, therefore without a main period. Finally there are also sources with a light-curve of too poor a $\mathrm{S} / \mathrm{N}$ ratio or a light-curve which slowly increases or decreases in the whole range of time. In the latter case the monitoring period was not long enough to detect periodicity; more data from the EROS or MACHO (Alcock et al. 1997) projects are certainly required. These last two groups of acceptable quality light-curves, because they indicate that the sources are variable but the light-curve is not good enough to assign a class of variability, correspond to about $20 \%$ of AGB stars. Table 1 summarizes the percentages of the different types of AGB stars found in this work and estimated as described in Sect. 5 .

\section{Spectral classification}

The spectrum of O-rich AGB stars is dominated in the visible wavelength range by $\mathrm{TiO}$ and $\mathrm{VO}$ molecular bands. We used these features to classify the observed sources as $\mathrm{O}-$ rich. In particular the $\mathrm{TiO}$ bands at $\lambda 4761$, $\lambda 4954, \lambda 5167, \lambda 5448, \lambda 5629, \lambda 5661, \lambda 5810, \lambda 5862, \lambda 6159$, $\lambda 6187, \lambda 6596$ (not always very deep) and $\lambda 7055 \AA$, and the VO bands at $\lambda 7400$ and $\lambda 7900 \AA$ were used. Stars which have approximately the same surface abundance of C and O, "S-stars", are characterized by the presence of molecular absorption bands of $\mathrm{ZrO}$ at $\lambda 5551, \lambda 5718$, $\lambda 5849, \lambda 6136, \lambda 6154, \lambda 6345, \lambda 6474, \lambda 6495$ and $\lambda 6933 \AA$, of YO at $\lambda 5972 \AA$, of TiO at $\lambda 7055 \AA$ (indicating probably a "MS" type) and of $\mathrm{LaO}$ at $\lambda 7404$ and $\lambda 7910 \AA$. We identify C-rich stars from the molecular absorption bands of CN around $\lambda 6925, \lambda 7088, \lambda 7225$ (triplets), $\lambda 5730$, $\lambda 5992, \lambda 6206, \lambda 6332, \lambda 6478, \lambda 6631, \lambda 6656$ and $\lambda 7437 \AA$, and of $\mathrm{C}_{2}$ at $\lambda 4737, \lambda 5165$ and $\lambda 5636 \AA$. The observed sample of 126 stars contains $33 \mathrm{C}$-rich stars, $7 \mathrm{~S}$ stars and $86 \mathrm{O}$-rich stars. Figure 3 shows the distribution of these sources in the color-magnitude diagram $\left(J-K_{\mathrm{S}}\right.$, $\left.K_{\mathrm{S}}\right)$. Vertical lines indicate the color which statistically discriminate between $\mathrm{O}$-rich and $\mathrm{C}$-rich stars. $\mathrm{TiO}$ and VO molecular bands, that dominate the spectrum of $\mathrm{O}$-rich AGB stars, are stronger in the $I$-band than in

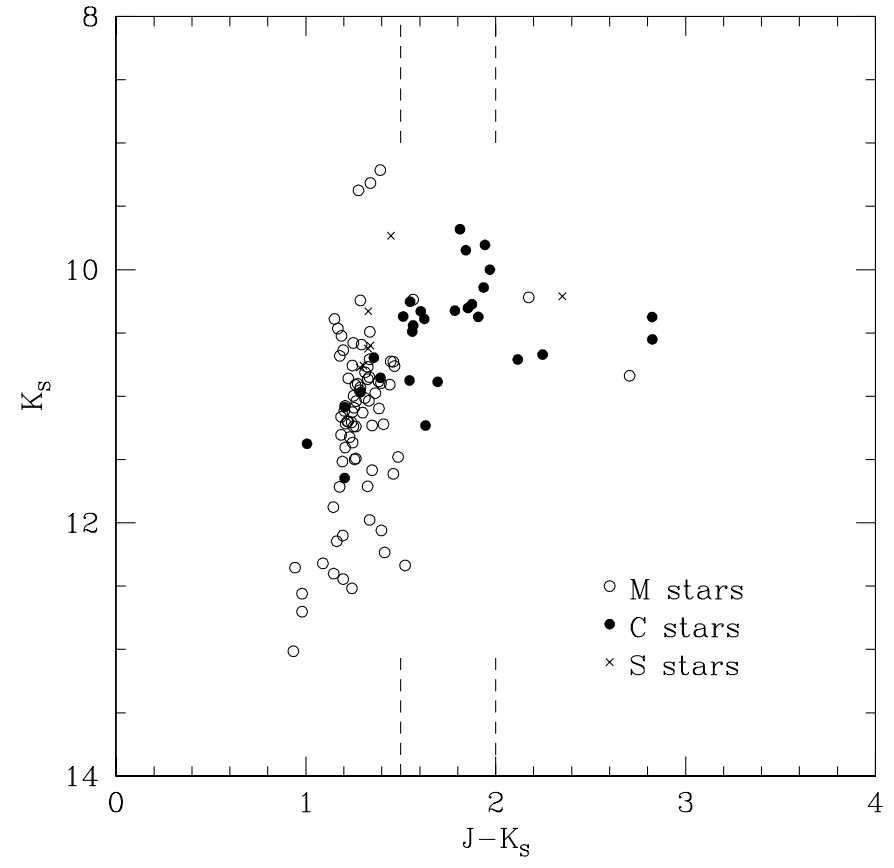

Fig. 3. Color-magnitude diagram of the spectroscopically observed DENIS candidates. Filled circles represent C-rich stars, empty circles represent $\mathrm{O}$-rich stars and crosses represent $\mathrm{S}$ stars. The vertical dashed lines discriminate between the regions dominated by C-rich, O-rich stars and obscured stars, as derived in Sect. 4.

the $J-$ and $K_{\mathrm{S}}$-bands; their $\left(J-K_{\mathrm{S}}\right)$ colors is almost constant while the bands become deeper with increasing $\mathrm{M}$ type, and with increasing $(I-J)$. $\mathrm{CN}$ and $\mathrm{C}_{2}$ molecular bands, that dominate the spectrum of C-rich AGB stars, are similarly stronger in the $I$ - and $J$-bands than in the $K_{\mathrm{S}}$-band. These stars cover a large range of $\left(J-K_{\mathrm{S}}\right)$ colors; it is not yet clear if this is correlated with the carbon spectral subtype. Objects with $\left(J-K_{\mathrm{S}}\right)>2.0$ can equally be $\mathrm{O}$-rich or $\mathrm{C}$-rich stars; relatively massive and luminous O-rich AGB stars with high mass-loss rates have been discovered in the Clouds (Wood et al. 1992; Zijlstra et al. 1996; van Loon et al. 1999).

For a detailed description of the features characterizing these stars and their variation as a function of the spectral sub-type we refer the reader to the Atlas of Digital Spectra of Cool Stars (Turnshek et al. 1985).

\section{Statistics}

All DCMC sources with few exceptions have an EROS counterpart, because EROS is deep enough $(I \approx 20 \div$ $21 \mathrm{mag})$ to detect the sources revealed at least in two DENIS wave bands. Not all identified DCMC sources are variables. This allows us to estimate what fraction of the AGB stars, present in the DCMC, are variables within the EROS completeness limit. For statistical purposes we focused on four quadrants (equivalent to one CCD area) where all DCMC sources were successfully cross-identified with EROS sources, areas that therefore have not been 

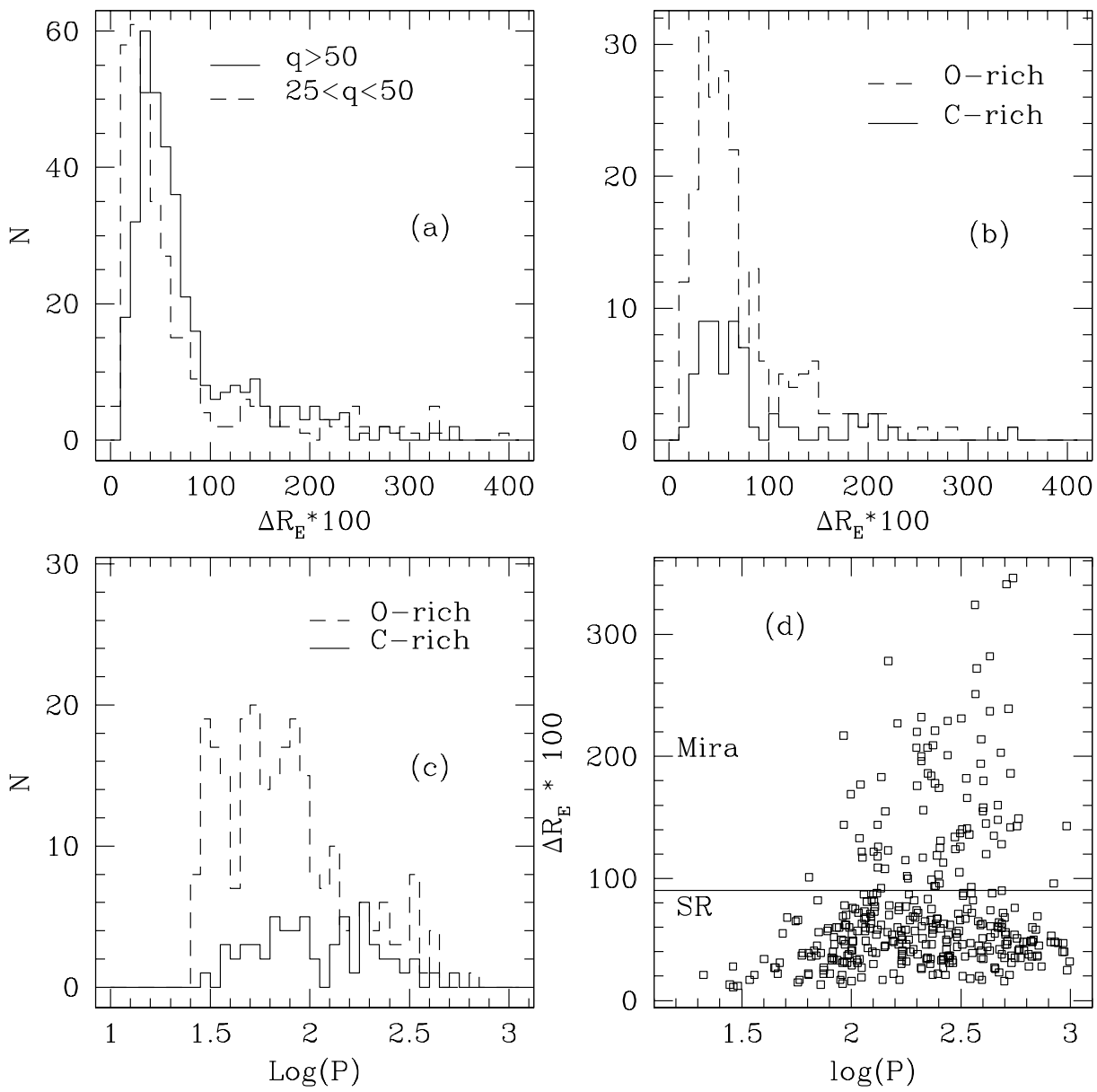

Fig. 4. Histogram distribution of the amplitude of a) all the variable sources. For good-quality light-curves both b) the distribution of amplitudes and c) periods are shown as a function of O-rich or C-rich type. d) shows how the amplitudes distribute as a function of $\log (P)$, the horizontal line indicates the applied selection criterion to distinguish Miras from $S R$ s.

affected by technical problems. In each quadrant there are on average 1500 DCMC sources of which we identify 100 to be in the AGB phase, i.e. $K_{\mathrm{S}}<12.0$ and $\left(J-K_{\mathrm{S}}\right)>1.2$.

Eight $\%$ of the all cross-identified sources show signs of variability in a good $(q>50)$ or acceptable $(25<q<50)$ light-curve. Among the AGB stars, as defined above, $65 \%$ are variable $(q>25)$. Changing the limits to characterize the location of the AGB stars in the color-magnitude diagram we obtain: $50 \%$ for $\left(J-K_{\mathrm{S}}\right)>1.0(61 \%$ if we also require $\left.K_{\mathrm{S}}<11.5\right), 65 \%$ for $\left(J-K_{\mathrm{S}}\right)>1.6,50 \%$ for $\left(J-K_{\mathrm{S}}\right)>2.4$. From the histogram of the distribution of the amplitudes of the detected variables (Fig. 4a) the EROS data are complete up to about $\Delta R_{\mathrm{E}}=0.3$.

\subsection{Amplitude of variability}

Figure 4 a shows the distribution of the amplitude of the 334 variable sources detected at least in $K_{\mathrm{S}}$. The peak of the distribution is around $\Delta R_{\mathrm{E}}=0.5 \mathrm{mag}$ for the sources with a good-quality light-curve, classified and shown in Fig. 5. Variable sources without a well defined period (irregular, poor $\mathrm{S} / \mathrm{N}$ or monotonic) peak at about $\Delta R_{\mathrm{E}}=0.3$. These peak positions roughly indicate the completeness of the EROS data for the chosen DCMC sample. Figure $4 \mathrm{~b}$ shows the amplitude distribution of the good-quality light-curve sources distinguishing between $\mathrm{C}$-rich and $\mathrm{O}-$-rich type. There is no apparent indication of a difference in amplitude between the two types. Figure 4c shows the period distribution also as a function of spectral type. O-rich AGB stars dominate at $\log (P)<2.0$, while $\mathrm{O}$-rich and $\mathrm{C}$-rich AGB stars are equally abundant at $\log (P)>2.0$. On average O-rich AGB stars in this sample tend to have shorter period than $\mathrm{C}$-rich stars; the latter have an almost constant distribution for $1.5<\log (P)<2.5$. Finally Fig. 4d shows the distribution of the amplitudes as a function of $\log (P)$ and the separation between Miras and Semi-Regulars in our sample.

\section{Discussion}

Figure 5 presents the $K_{\mathrm{S}}$ magnitude as a function of $\log (P)$ for the sources with a good-quality light-curve. We ignore the cepheids in the following discussion. Long period variables occupy the region of $\log (P)>1.4$. Three well defined groups of objects delineate three sequences of increasing magnitude with increasing period 
labelled $B, C$ and $D$ reflecting the correspondence with Wood et al. (1999): SRas are mostly distributed in relation $B$, Miras and those $S R a$ s that have $\Delta R_{E R O S}>0.7$ are all concentrated in relation $C$ and $S R b$ s (they have two periods) occupy relations $B$ and $D$.

The location of Miras is consistent with the LMC Mira sequence of Feast (1989). The distribution of SRas is also consistent with what has been found in the Galaxy (Bedding \& Zijlstra 1998) and previously in the Magellanic Clouds (Wood \& Sebo 1996). The longer period of the $S R b$ s gives rise to a new relation so far only discussed by Wood $(1999,2000)$, his sequence $D$.

There are few more objects in Fig. 5 with short periods that correspond to a sequence found by Wood and labelled by him as $A$. Both Wood's sequences $A$ and $B$ are densely populated at fainter magnitudes, while our sequences are better populated at brighter magnitudes. We attribute this difference to the limiting magnitude of DENIS (Cioni et al. 2000) and to the incompleteness of EROS in detecting small amplitude variables. The sequence of shorter periods of $S R b \mathrm{~s}$, confirmed to be mostly on sequence $B$, coincides also with the short period stars found in the Baade's window by the ISOGAL and MACHO collaboration (Alard et al. 2000). Wood's additional sequence $E$ of eclipsing binaries is definitely below the DENIS limiting magnitudes.

It is important to emphasize that the $K_{\mathrm{S}}$ magnitude of DENIS is a single epoch measurement and thus the $\log (P)$-magnitude diagram is affected by the scatter due to the amplitude of variation of each source; this effect is larger for Miras than for Semi-Regulars.

Each period-luminosity relation corresponds, theoretically, to a region of instability which is normally associated to a certain mode of pulsation; the presence of multiperiods indicates the coexistence of two pulsation modes. Figure 5 of Wood \& Sebo (1996) indicates that the long period in $S R b$ s results from radial fundamental mode pulsation, while Miras are first overtone pulsators and other Semi-Regulars are mostly high radial overtone pulsators; the predicted gap between fundamental and first overtone sequences is in very good agreement. This would also agree with the pulsation mode derived by Whitelock \& Feast (2000) from measured diameters (Hipparcos data) of Miras in the Milky Way. On the other hand if Miras are fundamental mode pulsators, as suggested by Wood et al. (1999), then the long period sequence of $S R b$ s requires another explanation, because theoretically there exists no pulsation mode at a longer period than that of the fundamental mode.

The presence of two simultaneous periods in $S R b$ s may be explained by the coupling between dust formation and star pulsation (Winters et al. 1994) because of a pulsation of longer period produced by the dynamical structure of the circumstellar dust shells. As shown by Fleischer et al. (1992) for large carbon to oxygen ratio $(\mathrm{C} / \mathrm{O}>1.8)$ the periodic ejection of a dust shell occurs exactly with the pulsational period while for $\mathrm{C} / \mathrm{O}<1.5$, as the amount of dust that can condense is smaller, shell

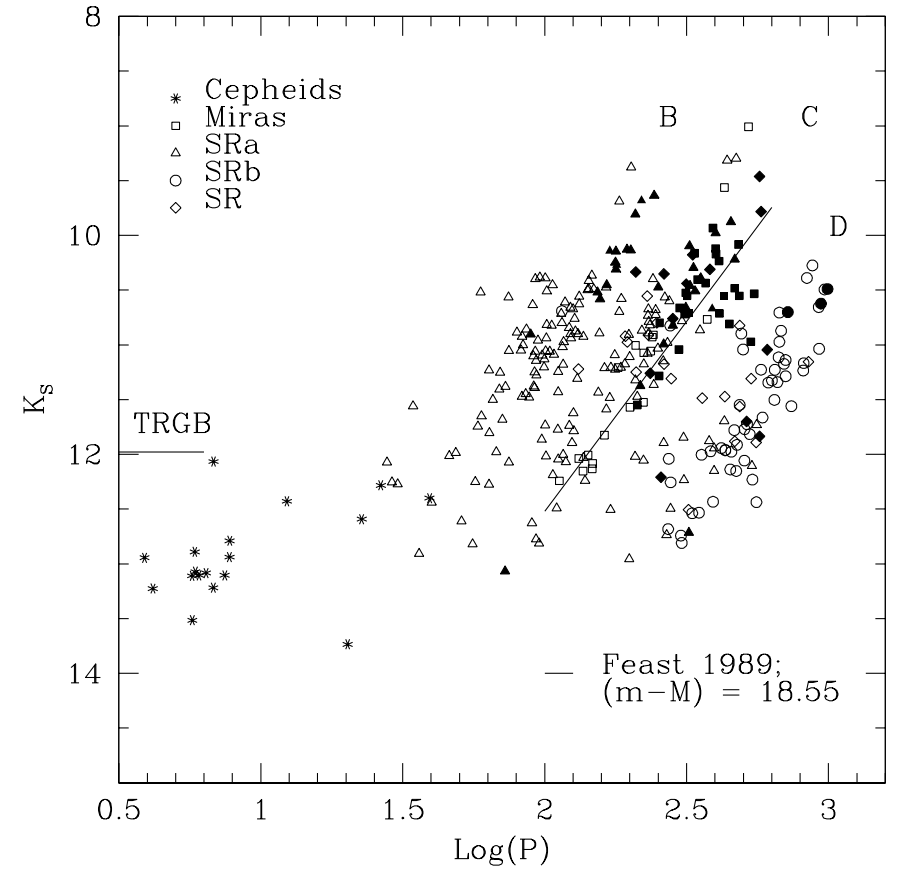

Fig. 5. Period-magnitude diagram for the good-quality lightcurve variable stars; the period $(P)$ is in days. Symbols refer to different types of variable sources and are explained in the plot. Only the long period of $S R b$ s is plotted in this figure, see Fig. 6 for the location of the two derived periods. Filled symbols are C-rich objects $\left(J-K_{\mathrm{S}}>1.5\right)$ and empty symbols are $\mathrm{O}-$ rich objects. The position of the tip of the red giant branch (TRGB) as derived by Cioni et al. (2000a) is indicated. The tilted line represents the period-luminosity relation derived by Feast (1989) for Miras in the Galaxy translated in the LMC at $(m-M)=18.55$. Capital letters identify the sequences as in Wood et al. (1999).

ejection takes place at longer intervals than one period (Fleischer et al. 1999): the pulsation corresponds to the shorter of the two periods and the longer is due to obscuration by dust. It is however questionable whether such a model applies to stars with silicate type dust and lower mass-loss rates $\left(10^{-6}\right.$ to $\left.10^{-8} M_{\odot} \mathrm{yr}^{-1}\right)$ such as those derived from CO observations (Loup et al. 1993), but preliminary calculations support that the model does apply (Winters, private communication). If there is circumstellar dust the star is expected to have redder $\left(J-K_{\mathrm{S}}\right)$ than those observed by DENIS in this sample. On the other hand if at these colours the molecular blanketing effect (in the $J$ band due to $\mathrm{C}_{2}$ and $\mathrm{CN}$ ) dominates the dust obscuration effect the blue $\left(J-K_{\mathrm{S}}\right)$ colour is explained.

Wood et al. $(1999,2000)$ suggest that sequence $D$ is populated by the longer period of a binary system. Such a scenario is also poorly constrained. We found in our sample only three possible eclipsing binaries (Fig. 6). Moreover the number of objects populating this sequence is too high with respect to theoretical predictions. Whitelock (1986) shows, from measurements in globular clusters, that the evolutionary path of long period variables goes from $S R a$ to Mira following a line of shallower slope, in agreement 


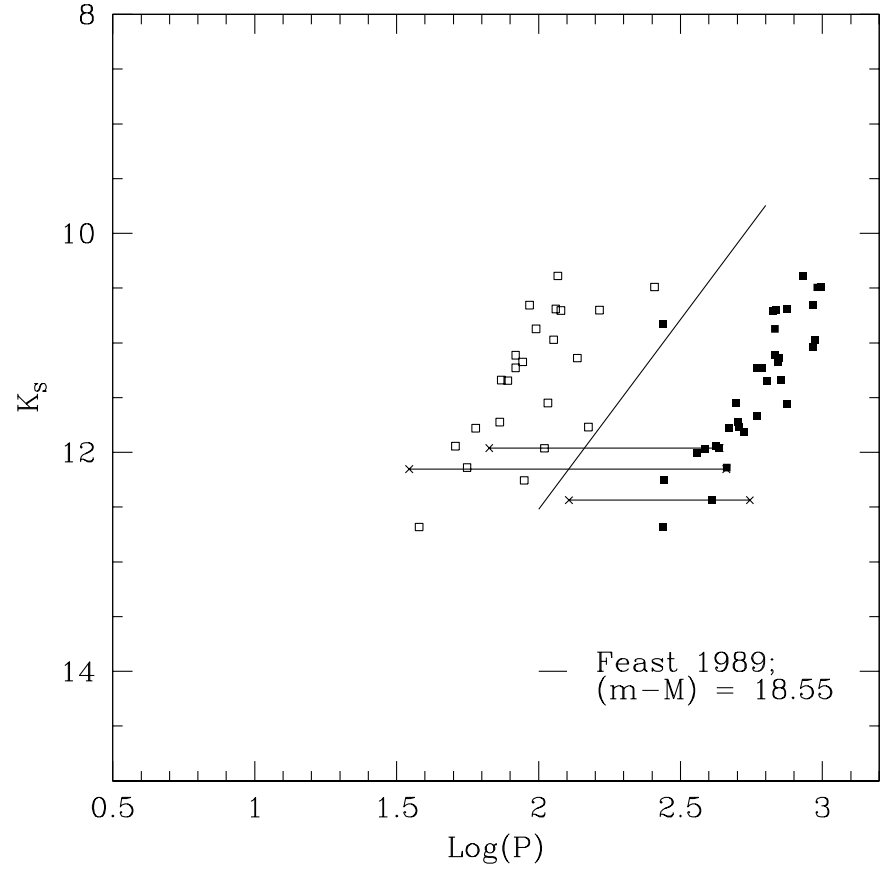

Fig. 6. Period-magnitude diagram of $S R b$ s. Filled symbols show the long period and empty symbols show the short period for the same source. Horizontal lines connect possible eclipsing binaries. The period $(P)$ is in days.

with the theoretical calculations by Vassiliadis \& Wood (1993), than the period-magnitude relation. The evolutionary path thus shows that the stars pass through a variable phase, then the pulsation goes away and they slowly move into the next sequence. Gaps between the sequences reflect a "phase" of some stability which could be represented by the $35 \%$ of non-variable AGB stars. However because of the existing gap between the starting of the core Helium burning (TRGB) and the beginning of the TP-AGB phase (Iben \& Renzini 1983) some of the AGB stars in our sample might be in an earlier evolutionary phase (E-AGB) or they are TP-AGB stars in between thermal pulses that esperience a significant decline of luminosity (Wood et al. 1999). Little evidence of pulsation has been found in AGB stars just above the horizontal branch (Feast \& Whitelock 1987), these E-AGB stars might be of the same type as those AGBs we do not detect, within the completness limits of the sample, as variables. Alard et al. (2000) found that $92 \%$ of the stars in their sample are variable. These stars have been selected to have a detection both at [7] $\mu \mathrm{m}$ and [15] $\mu \mathrm{m}$ (ISO bands). Because of the detection at [15] $\mu \mathrm{m}$, that probes the existence of a circumstellar dust envelope (Omont et al. 1999), we think that Alard et al. sample is formed by TP-AGB stars which are therefore more evolved than our sample of AGB stars selected on the basis of nearinfrared colours. It is thus not surprising that we find a lower percentage of AGB variables. However it is remarkable that in our sample only $50 \%$ of the high mass-loss stars $\left(\left(J-K_{\mathrm{S}}\right)>2.4\right)$ are variable, because mass-loss and variability are believed to be connected. The result may be due to incompleteness of the sample (Sect. 5.1); note that the sample size for stars with $\left(\left(J-K_{\mathrm{S}}\right)>2.4\right)$ is much smaller because DENIS does not detect very obscured AGB stars.

The color-magnitude diagrams $\left(J-K_{\mathrm{S}}, K_{\mathrm{S}}\right)$ shown in Fig. 7 characterize the location of the different types of variable. Miras cover uniformly the region where $\mathrm{O}$-rich, C-rich and obscured stars are located (Fig. 3). Comparing the four diagrams, the reddest objects are all of Mira type. Not all Miras, however, have red colors. This may indicate a difference in initial mass. The initial "position" (luminosity, period, color) of the stars in the TP-AGB phase is given when they experience the first TP. All TP-AGB stars loose mass at a rate that varies from $10^{-8}$ to $10^{-4} M_{\odot} \mathrm{yr}^{-1}$ and will end as a $0.6 \div 1.0 M_{\odot}$ white dwarf. Because the time on the AGB of the less massive stars is much longer than that of the more massive stars, the mass loss rate of a low mass star can be much lower. As a consequence in the last stages of the AGB (Mira) low mass stars do not become much redder because of mass loss, but the more massive stars will. The AGB phase is so brief (Vassiliadis \& Wood 1993) that there is very little increase in luminosity. Stars that are too light will remain O-rich because the TPs have too little effect. Stars that are too massive will remain O-rich because of hot-bottom burning. Only stars in a well-defined mass interval will become C-rich. In the LMC this explains why C-rich stars have almost all the same luminosity (Groenewegen 1994). SRas are distributed in both branches of O-rich and Crich stars while $S R b$ s are mostly concentrated only in the O-rich branch (Fig. 7). Sources with good or acceptable quality light-curves, but no classification, are distributed in different regions of the diagram and their type can probably be derived from the diagrams of the classified sources.

$S R b$ s might be in a transition phase between O-rich SRas and Miras. At the beginning and at the end of the TP-AGB phase the amount of material available to form dust is high, in the first case because the excess of oxygen is high and in the other one because the excess of carbon is high. We expect such objects to have light-curves like SRas; dust would obscure the star at intervals approximately equal to the pulsation period. In the transition from $\mathrm{O}$-rich to $\mathrm{C}$-rich stars the amount of dust reduces, therefore these objects would behave like $S R b s$. Another possibility is that $S R b$ s are less evolved than O-rich SRas and have therefore have less matter that can condense into dust, main requirement to have a multiperiodic light-curve such as those calculated by Winters et al. (1994). The monitoring of dust features for SRas and $S R b$ s of comparable near-infrared properties will certainly put constraints on the proposed scenarios and allow to discriminate against the existence of a binary system; such a program is currently under development.

Comparing the $\log (P)$-magnitude diagram with the models by Vassiliadis \& Wood (1993) we find that our sample contains AGB stars with masses in the range from 0.9 to $5 M_{\odot}$ and age range from 0.1 to $10 \mathrm{Gyr}$. 

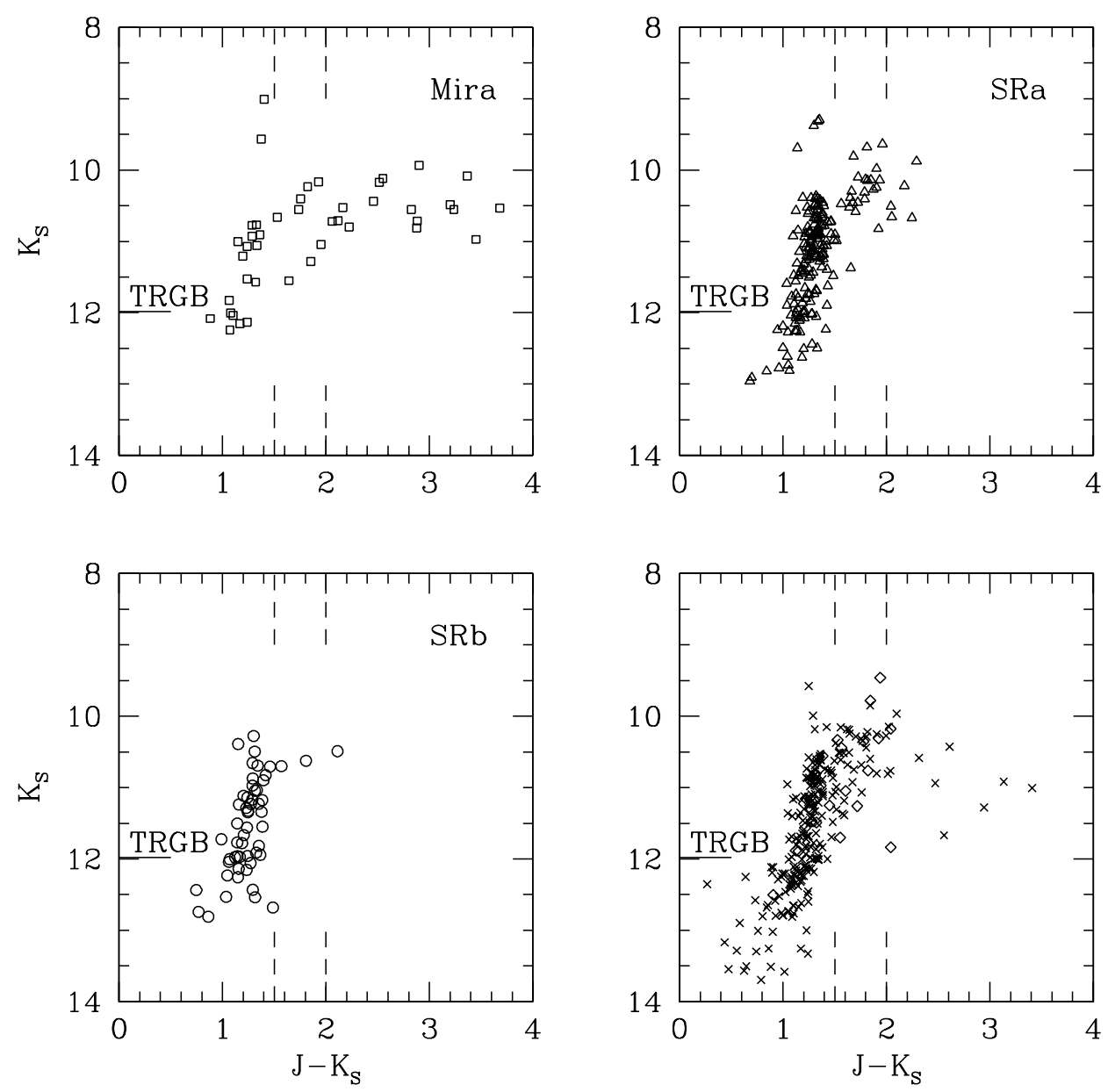

Fig. 7. Color-magnitude diagrams $\left(J-K_{\mathrm{S}}, K_{\mathrm{S}}\right)$ of the detected variables. Symbols are as in Fig. 5 and crosses indicate sources of acceptable-quality light-curve; Cepheids are excluded from these plots.

An intermediate/old population is definitely present in the LMC.

It is interesting to notice that there are some SemiRegulars and few Miras located below the TRGB. These sources could be AGB stars in the early evolutionary phase (burning $\mathrm{He}$ in a shell) or TP-AGB (burning $\mathrm{H}$ and $\mathrm{He}$ into concentric shells) between thermal pulses or variables for the first time on the red giant branch. We are going to investigate spectroscopically the nature of these variable objects.

\section{Summary}

Based on the cross-identification between the DCMC and the EROS data, in a region containing the OC field in the bar of the LMC, we have classified the best detected variables and shown three $\log (P)-K_{\mathrm{S}}$ relation for red giant variables. Combined spectroscopic observations have shown the distinction between $\mathrm{C}$-rich and O-rich AGB stars in the color-magnitude diagram $\left(J-K_{\mathrm{S}}, K_{\mathrm{S}}\right)$. C-rich stars occupy the upper position of the $\log (P)-K_{\mathrm{S}}$ relations. We have confirmed three of the relations found by Wood using MACHO data. The nature of the relation at the longest period is still unknown and further observational investigations are necessary and under development.

Acknowledgements. The access to the EROS database has been kindly granted by the EROS collaboration. The GAIA and CURSA packages have been available thanks to the work of the Starlink project, UK. We thank Jean-Philippe Beaulieu, Sylvain Lupone and Gregoor Verschuren for valuable assistance during data treatment, Ariane Lançon, Yvonne Simis, Peter Wood, Jan-Martin Winters Joris Blommaert and Patricia Whitelock for useful discussions.

\section{Appendix A: Tables}

Table A.2 listing the DENIS/EROS sources (Fig. 5) with a good light-curve quality, classified in the present work with observed spectra is electronically available at the CDS. It contains the following items: the DCMC source identifier, the EROS source number, the period, the lightcurve quality factor, the classification as described in Table A.1, the EROS quadrant where the source was observed and notes that could be the Blanco et al. (1980) counterpart or the longer period in the case of $S R b s$.

Also, a Table A.3 listing the spectral nature of the newly observed DENIS sources, not included in Fig. 5 and 
Table A.1.

\begin{tabular}{rl}
\hline Value & Description \\
\hline 0 & Cepheid \\
11 & Mira \\
13 & Mira with bump \\
15 & $S R a$ \\
16 & $S R a$ with bump \\
19 & $S R$ \\
2 & $S R b$ \\
\hline
\end{tabular}

in the previous table, is available at the same place. A more detailed analysis with the aim to assign the spectral type to these sources will be the subject of a forthcoming paper, together with equivalent observations in other locations in the LMC and in the Small Magellanic Cloud.

\section{References}

Alard, C., Blommaert, J., Cesarsky, C., et al. 2000, ApJ [astro-ph/0101094]

Alcock, C., Allen, W. H., Allsman, R. A., et al. 1997, ApJ, 474, 217

Ansari, R. 1996, Vistas Astron. 40, 519

Bedding, T. R., \& Zijlstra, A. A. 1998, ApJ, 506, 47

Blanco, V. M., McCarthy, M. F., \& Blanco, B. M. 1980, ApJ, 242,938

Cioni, M. R., van der Marel, R. P., Loup, C., et al. 2000b, A\&A, 359, 601

Cioni, M. R., Loup, C., Habing, H. J., et al. 2000a, A\&AS, 144,235

Cohen, J. G., Persson, S. E., Elias, J. H., et al. 1981, ApJ, 249, 481

Eggen, O. J. 1975, AJ, 195, 661

Epchtein, N., Deul, D., Derriere, S., et al. 1999, A\&A, 349, 236

Feast, M. W., Glass, I. S., Whitelock, P. A., \& Catchpole, R. M. 1989, MNRAS, 241, 375

Feast, M. W., \& Whitelock, P. A. 1987, in Late Stages of Stellar Evolution, ed. S. Kwok, \& S. R. Pottasch (Reidel), 33
Feast, M. W. 1963, MNRAS, 125, 27

Fleischer, A. J., Gauger, A., \& Sedlmayr, E. 1992, A\&A, 266, 321

Fleischer, A. J., Winters, J. M., \& Sedlmayr, E. 1999, in AGB Stars, IAU Symp., 191, 187

Glass, I. S., \& Lloyd Evans, T. 1981, Nature, 291, 303

Groenewegen, M. A. T., \& de Jong, T. 1994, A\&A, 288, 782

Groenewegen, M. A. T., \& Whitelock, P. A. 1996, MNRAS, 281,1347

Hughes, S. M. G. 1989, AJ, 97, 1634

Kerschbaum, F., \& Hron, J. 1992, A\&A, 263, 97

Kholopov, P. N. 1985 in General Catalogue of Variable Stars (1) (Moscow Nauka Pub. House)

Iben, I. Jr., \& Renzini, A. 1983, A\&AR, 21, 271

Le Bertre, T. 1992, A\&AS, 94, 377

Loup, C., Forveille, T., Omont, A., \& Paul, J. F. 1993, A\&AS, 99, 291

Loup, C. 1998, in New View of the Magellanic Clouds, IAU Symp., 190, 35

Omont, A., Ganesh, S., Alard, C., et al. 1999, A\&A, 348, 755

Schwarzenberg-Czerny, A. 1999, ApJ, 516, 315

Turnshek, D. E., Turnshek, D. A., Craine, E. R., \& Boeshaar, P. C. 1985, in An Atlas of digital spectra of cool stars

van Loon, J. Th., Groenewegen, M. A. T., de Koter, A., et al. 1999, A\&A, 351, 559

Vassiliadis, E., \& Wood, P. A. 1993, ApJ, 413, 641

Whitelock, P. A. 1986, MNRAS, 219, 525

Whitelock, P. A., \& Feast, M. 2000, MNRAS, 319, 759

Winters, J. M., Fleischer, A. J., Ganger, A., et al. 1994, A\&A, 290,623

Wood, P. R., Whiteoak, J. B., Hughes, S. M. G., et al. 1992, ApJ, 397, 552

Wood, P. R. 2000, PASA, 17, 18

Wood, P. R., Alcock, C., Allsman, R. A., et al. 1999, IAU Symp., 191, 151

Wood, P. R., \& Sebo, K. M. 1996, MNRAS, 282, 958

Wood, P. R., Bessel, M. S., \& Paltoglou, G. 1985, ApJ, 290, 477

Zijlstra, A. A., Loup, C., Waters, L. B. F. M., et al. 1996, MNRAS, 279, 32 